\title{
LINEAR DIFFERENTIAL EQUATIONS WITH PERIODIC COEFFICIENTS
}

\author{
T. A. BURTON
}

1. Introduction. We consider a system of linear differential equations

$$
X^{\prime}=A(t) X \quad\left({ }^{\prime}=d / d t\right)
$$

where $X$ is an $n$ dimensional column vector and $A(t)$ is an $n \times n$ matrix whose elements are continuous periodic functions of a real variable $t$.

Epstein [2] has shown that if $A(t)$ is periodic and odd then all solutions of (1) are periodic. Also, using formulae from differential geometry, Epstein obtained a necessary condition that all solutions of (1) be periodic provided that $A(t)$ is $3 \times 3$, skew symmetric, and periodic.

We show that if $A(t)$ is skew symmetric and periodic, then every solution of (1) is almost periodic. This theorem is important for two reasons. First, it is of interest in itself. Second, Epstein has shown that the solutions of (1) depend on those of two systems, one of which is symmetric and the other skew symmetric. The coefficients of the symmetric system will be periodic if the solutions of the skew symmetric system are periodic with the same period as the original system. Since the fundamental solution matrix of (1) can be expressed as $\tilde{X}(t)=P(t) Y(t)$ where $P(t)$ is periodic and $Y(t)=\exp D t$ is the fundamental solution matrix of $Y^{\prime}=D Y$ with $D$ constant, one would be reluctant to use Epstein's technique of separating (1) into two systems unless he could be sure that both of the resulting systems would have solutions of a correspondingly simple form as that of (1). Our theorem enables us to show that the fundamental solution matrix of the symmetric system can be expressed as $F(t) \exp D t$ where $F(t)$ is almost periodic and $D$ is constant.

2. Almost periodic solutions. Let us denote $X$ by $\operatorname{col}\left(x_{1}, \cdots, x_{n}\right)$.

THEOREM 1. If $A(t)$ is periodic and skew symmetric, then all solutions of (1) are almost periodic.

Proof. The scalar function $V=x_{1}^{2}+\cdots+x_{n}^{2}$ is a Liapunov function for (1) and $d V / d t \equiv 0$ along trajectories of (1). Hence the surface defined by $V=$ const. contains all solutions which start on it and so every solution vector of (1) has constant euclidean length. Since

Received by the editors July 1, 1965. 
$A(t)$ is periodic there exists a Liapunov transformation $X=L(t) H$ mapping (1) into

$$
H^{\prime}=B H
$$

where $L(t)$ is an $n \times n$ periodic matrix with $L(m T)=I$ (here, $m$ is any integer, $I$ is the identity matrix, and $T$ is the period of $A(t))$ and $B$ is a constant $n \times n$ matrix (see Gantmacher [3]). If $H(t)$ is the fundamental solution matrix of $H^{\prime}=B H$, then $\tilde{X}(t)=L(t) H(t)$ is the fundamental solution matrix of (1). Thus, $\tilde{X}(m T)=H(m T)$. Let $X_{1}(t)$, $\cdots, X_{n}(t)$ and $H_{1}(t), \cdots, H_{n}(t)$ be the column vectors of $\tilde{X}(t)$ and $H(t)$ respectively. Then $X_{i}(m T)=H_{i}(m T)$ and $X_{i}(m T)$ has constant euclidean length so the same is true for $H_{i}(m T)$. Since $L(t)$ is nonsingular the characteristic roots of $B$ have real parts which are zero and each characteristic root of $B$ is simple. For if some characteristic root had a nonzero real part, then the corresponding solution would become either unbounded or it would tend to zero and both of these are impossible since the solutions have constant euclidean length. Also, if some characteristic root (with zero real part) were not simple, then there would be a solution which becomes unbounded and this again is impossible. Now to each of these (purely imaginary or zero) characteristic roots $\alpha_{i}$ of $B$ there corresponds a solution of the form $c_{i} \exp \left(\alpha_{i} t\right)$ where $c_{i}$ is a constant vector; thus the $H_{i}(t)$ are periodic and hence $\tilde{X}(t)$ is almost periodic [1].

3. The form of the solutions. We now suppose that $A(t)$ is periodic but not skew symmetric. Epstein shows that $\tilde{X}(t)=Z(t) W(t)$ where $Z(t)$ is the fundamental solution matrix of

$$
Z^{\prime}=1 / 2\left[A(t)-A^{T}(t)\right] Z
$$

and $W(t)$ is the fundamental solution matrix of

$$
W^{\prime}=1 / 2\left[Z^{T}(t)\left(A(t)+A^{T}(t)\right) Z(t)\right] W .
$$

Since $A(t)$ is periodic, $A-A^{T}$ is skew symmetric and periodic. Thus, by Theorem 1 , it follows that $Z(t)$ is almost periodic and the coefficient matrix of (4) is almost periodic.

Theorem 2. There exist matrices $F(t)$ and $D$ where $F(t)$ is almost periodic and $D$ is constant such that $W(t)=F(t) \exp D t$.

Proof. From the proof of Theorem 1 we have $Z(t)=L(t) K(t)$ where $L(t+T)=L(t)$ and the elements of $K(t)$ are periodic of some unknown period. Also, it is known that $\tilde{X}(t)=P(t) \exp D t$ where $D$ is constant and $P(t+T)=P(t)$. Since $\tilde{X}(t)=Z(t) W(t)$ we have $P(t) \exp D t=$ 
$L(t) K(t) W(t)$ or $W(t)=K^{-1}(t) \mathrm{L}^{-1}(t) P(t) \exp D t$. But $K^{-1}(t) L^{-1}(t) P(t)$ is almost periodic and so $W(t)=F(t) \exp D t$ where $F(t)$ is almost periodic.

Added in Proof. It seems that Theorem 1 is known. See Amer. Math. Monthly 71 (1964) 774-776. This reference was pointed out by J. D. Schur.

\section{REFERENCES}

1. A. Besicovitch, Almost periodic functions, Cambridge Univ. Press, Cambridge, 1932; p. 6.

2. I. Epstein, Periodic solutions of systems of differential equations, Proc. Amer. Math. Soc. 13 (1962), 690-694.

3. F. Gantmacher, Applications of the theory of matrices, Interscience, New York, 1959, 140-143.

University of Alberta, Edmonton 